\title{
Oncologic outcomes of radical nephroureterectomy (RNU)
}

\author{
Alexander P. Kenigsberg ${ }^{1}$, Xiaosong Meng ${ }^{1}$, Rashed Ghandour ${ }^{1}$, Vitaly Margulis ${ }^{1,2}$ \\ ${ }^{1}$ Department of Urology, University of Texas Southwestern Medical Center, Dallas, TX, USA; ${ }^{2}$ Institute for Urology and Reproductive Health, \\ Sechenov University, Moscow, Russia \\ Contributions: (I) Conception and design: All authors; (II) Administrative support: AP Kenigsberg, X Meng, R Ghandour; (III) Provision of study \\ materials or patients: AP Kenigsberg; (IV) Collection and assembly of data: AP Kenigsberg, X Meng; (V) Data analysis and interpretation: None; (VI) \\ Manuscript writing: All authors; (VII) Final approval of manuscript: All authors. \\ Correspondence to: Vitaly Margulis, MD. Department of Urology, University of Texas Southwestern Medical Center, 2001 Inwood Rd, WCBE3, $4^{\text {th }}$ \\ Floor, Dallas, TX 75390-9110, USA. Email: vitaly.margulis@utsouthwestern.edu.
}

\begin{abstract}
Radical nephroureterectomy is the mainstay of surgical treatment for upper tract urothelial carcinoma (UTUC), a disease which comprises approximately $5 \%$ of urothelial malignancies. Minimallyinvasive and nephron-sparing interventions have been explored, although thus far have not shown comparable oncologic outcomes except in a relatively narrow set of patients. Due to the relative rarity of the disease, it has taken decades and multi-disciplinary efforts to sufficiently identify prognostic factors of oncologic outcomes. Despite these efforts, however, oncologic outcomes of nephroureterectomy have remained remarkably stable over the past 30 years. New techniques, such as laparoscopic and robotic surgery, have been applied to this procedure. High level evidence regarding equivalent oncologic outcomes is lacking and open surgery remains the standard of care for high-stage disease, although there is a role for laparoscopic and robotic nephroureterectomy. The importance of bladder cuff removal in improving oncologic outcomes has been broadly accepted, although there is no consensus as to the most oncologically appropriate technique. There does appear to be evidence that endoscopic techniques confer worse oncologic control. The role of lymphadenectomy remains controversial, although there is evidence that increased nodal yield could have oncologic benefit. Given disease heterogeneity and varied technical approaches to the procedure, no consensus standardized template has been identified. There is level 1 evidence for the use of intravesical chemotherapy peri-operatively and that this intervention can improve the risk of intravesical recurrence. Advances in systemic neoadjuvant and adjuvant chemotherapy have yielded promising results and are likely to become standard of care for patients without contraindications. Immunotherapy and targeted biologic agents are also likely to improve the surgical efficacy of radical nephroureterectomy as well. Ultimately, more high level evidence is needed to identify successful surgical and medical approaches to UTUC and multiinstitutional collaboration is critical to this progress.
\end{abstract}

Keywords: Nephroureterectomy; robotic surgery; laparoscopic surgery; neoadjuvant chemotherapy

Submitted Oct 05, 2019. Accepted for publication Dec 20, 2019.

doi: $10.21037 /$ tau.2019.12.29

View this article at: http://dx.doi.org/10.21037/tau.2019.12.29

\section{Introduction}

Upper tract urothelial carcinoma (UTUC) comprises approximately $5 \%$ of all urothelial malignancies (1) and between $5-10 \%$ of renal tumors (2). Nearly $60 \%$ of these tumors are found in the pelvicalyceal system and the remainder along the course of the ureter (3). The European
Association of Urology (EAU) guidelines on UTUC identify a select group of patients for whom minimally invasive endoscopic management is oncologically appropriate. This includes patients with a functioning contralateral kidney and low grade tumors that are unifocal, less than $1 \mathrm{~cm}$ in size, have no evidence of infiltration on delayed phase imaging, and for whom close follow up is feasible (2). 
Nearly two-thirds of patients with UTUC have high-grade disease. Given the preponderance of high-grade disease, the lack of reliable data confirming the oncologic safety of conservative management in this setting, and the limited validated selection criteria for endoscopic management, the vast majority of patients with UTUC require radical surgical resection of their disease. The gold-standard treatment for these patients is radical nephroureterectomy with excision of a bladder cuff (4). As the majority of patients with UTUC will undergo radical nephroureterectomy, it is important to understand the preoperative, intraoperative, and postoperative predictors and the effects of surgical techniques and approaches on oncologic outcomes.

In any critical appraisal of the literature, it is important to keep in mind the relative rarity of the disease and lack of level 1 evidence available. Much of this has been countered by the development of collaborative multi-institutional efforts. While this has contributed power to studies and allowed for the greater understanding of UTUC, it does mean that many of the studies overlap in the data evaluated with potential underlying data quality issues that may exist over years of multi-institutional data collection among heterogenous populations and practices.

\section{Radical nephroureterectomy vs. partial ureterectomy}

Partial or segmental ureterectomy has long been considered a possible alternative to radical nephroureterectomy in the treatment of UTUC, particularly prior to the development of flexible ureteroscopy. Mazeman was the first to compare oncologic outcomes of radical nephroureterectomy and distal ureterectomy with ureteral reimplantation and found comparable oncologic control (5). Johnson and Babaian described a case series in which distal ureterectomy was utilized for management of distal, low-grade noninvasive UTUC (6). In their series of six patients, only one patient had ipsilateral recurrence at 26 months, requiring radical nephroureterectomy. Zungri et al. reported on the outcomes of 73 patients treated between 1938 and 1985 who underwent radical nephroureterectomy or segmental ureterectomy and found no difference in oncologic outcomes for patients who underwent conservative treatment for superficial tumors, but worse oncologic outcomes for those who underwent conservative treatment than radical nephroureterectomy for pT2 tumors $(33.3 \%$ vs. 57.1\% 5-year survival, respectively) (7) and concludes, as other retrospective, population-level studies, and meta-analyses, that segmental ureterectomy may be reasonable in a select cohort (8-10). The largest study to evaluate this issue was a sub-group analysis of the UTUC Collaboration cohort, a multi-institutional initiative that collected data on over 1,300 patients from 12 academic centers (4). Their subgroup analysis (11) demonstrated comparable 5-year cancer-specific survival (CSS) for radical nephroureterectomy and partial ureterectomy $(72.1 \%$ vs. $67.5 \%$, respectively), although there was a higher rate of adjuvant chemotherapy utilization for the partial ureterectomy than radical nephroureterectomy cohort (25.9\% vs. $16.8 \%$, respectively).

All of the studies comparing segmental ureterectomy to radical nephroureterectomy are limited in their retrospective nature and unmatched cohorts. The aforementioned studies all acknowledge a selection bias and that those in the radical nephroureterectomy cohorts have higher grade and stage disease disproportionate to the segmental ureterectomy cohorts. No randomized control trials have been conducted to evaluate the true oncologic outcomes between these interventions. Further, there is a high risk of recurrence in UTUC when radical nephroureterectomy is not performed. Strong et al. noted a 30\% recurrence rate of UTUC at the ureteral stump in cases managed with segmental ureterectomy although proximal recurrences were also noted (12). Bagrodia et al. analyzing the UTUC collaborative data noted a similar $31.1 \%$ recurrence rate among those treated conservatively with high grade disease vs. $19.4 \%$ for those with low grade disease (11). In light of these data, radical nephroureterectomy is still considered the standard of care except in cases outlined by the EAU guidelines as amenable to conservative management. Further matched-cohort investigations are necessary to determine if segmental ureterectomy can be safely offered in an expanded number of patients.

\section{Patient predictors of oncologic outcomes}

Various patient factors have been identified and studied in efforts to understand oncologic outcomes of nephroureterectomy. While some are more modifiable than others, this information can be useful in the counseling of patients prior to nephroureterectomy.

\section{Age}

Age, as with many urothelial cancers, has been postulated to be predictive of cancer-specific mortality. Shariat et al. 
evaluated the role of age on cancer-specific outcomes of radical nephroureterectomy (13). Unsurprisingly these authors found decreased overall survival in patients over age 60 and decreased cancer specific survival for patients over 80 who underwent radical nephroureterectomy. These authors conclude, however, that it is difficult to attribute these outcomes to age alone, as patients over 70 were less likely to undergo lymphadenectomy or receive adjuvant chemotherapy. While other studies have found age to predict CSS, recurrence free survival (RFS), and OS (14), level 1 evidence is still lacking to demonstrate the role age plays on oncologic outcomes of radical nephroureterectomy.

\section{Gender}

Initially patient gender was considered to be a predictive factor of poor oncologic outcomes after nephroureterectomy, given known gender disparities in bladder cancer (15). Population level data from the Surveillance, Epidemiology, and End Results (SEER) database demonstrated that women present with higher stage and higher grade UTUC (16). These authors note, however, that when controlling for stage and grade disparities, gender is no longer predictive of cancer specific survival after nephroureterectomy. In a multi-institutional series of 754 patients treated with radical nephroureterectomy without neoadjuvant chemotherapy, Shariat et al. demonstrated no differences in cancer recurrence or cancer specific survival between men and women (17), which has been substantiated with subsequent multi-institutional analyses.

\section{Race}

Racial disparities have been noted in cancer-specific and recurrence-free survival of patients after radical nephroureterectomy. In an analysis of the SEER data, Raman et al. found significantly worse overall survival outcomes for black, non-Hispanic patients who underwent radical nephroureterectomy (18) and that this was an independent predictor of CSS on multivariable analysis. There is limited reported patient data on this topic and room for investigation into whether such racial disparities exist in UTUC treatment and can be overcome with improved detection of disease.

\section{Smoking status}

Current smoking status also has bearing on long-term oncologic outcomes following nephroureterectomy. Rink et al. evaluated the impact of smoking on disease progression and CSS and found that, despite current smokers being of significantly younger age within the cohort, current and heavy long-term smokers were at greater risk of advanced disease, higher likelihood of recurrence, and worsened CSS relative to non-smokers (19). These authors also noted a washout effect, whereby patients who had quit smoking greater than 10 years prior to nephroureterectomy did not demonstrate these disparities relative to nonsmokers. Xylinas et al. investigated the role of smoking on intravesical recurrence of urothelial carcinoma after radical nephroureterectomy (20). Similarly, these authors found that those with $>10$ years of abstinence from cigarettes had a lower rate of intravesical recurrence than current smokers and noted that a history of greater than 20 pack years of smoking conferred a much greater risk of intravesical recurrence.

\section{Weight}

Obesity has been implicated in poor oncologic outcomes following nephroureterectomy, as with multiple other malignancies, likely due to its induction of increased systemic inflammation. Ehdaie et al. noted a steep dropoff in 5-year RFS as stratified by weight of $84 \%$ for those with body mass index (BMI) $<25,65 \%$ with BMI 25-29.9, and $47 \%$ with BMI of 30 or greater (21). These authors also note that BMI $>30$ is an independent predictor of CSS. Subsequent analyses have further stratified out CSS and demonstrated a similar drop-off as with RFS (22).

\section{Operative factors}

\section{Timing of surgery}

The timing of nephroureterectomy, particularly as it relates to diagnostic procedures, has been evaluated as a potential prognostic indicator of oncologic outcomes. The issue of ureteroscopy prior to radical nephroureterectomy and the effect on oncologic outcomes is two-fold: the effect of delay in radical nephroureterectomy on outcomes and the effect of ureteroscopy itself on oncologic results. Boorjian et al. sought to investigate whether patients who underwent ureteroscopic biopsy had worse oncologic outcomes than those who had direct nephroureterectomy based on filling defects on imaging and cytology, due to delay to definitive management or potential tumor seeding (23). These 
authors found no difference in oncologic outcomes between the groups, despite mean time to nephroureterectomy of nearly 200 days. Sankin et al. similarly found no difference in CSS, metastasis-free survival, or overall survival between those who underwent prior ureteroscopy, although found a significantly increased risk of intravesical recurrence at 3 years (RFS: $71 \%$ with no prior ureteroscopy versus $42 \%$ with prior ureteroscopy) (24). As for timing, the EAU guidelines do not offer a specific recommendation, although they note that most authors propose between 30 days and 3 months after ureteroscopy. Sundi et al. retrospectively evaluated patients in an "early" surgical group (radical nephroureterectomy within 3 months of ureteroscopy) and "late" group and found 5-year CSS of $72 \%$ and $71 \%$, respectively (25). This finding is confounded, however, by the fact that the most common reason for delayed surgery was administration of neoadjuvant chemotherapy, which could affect CSS. Xia et al. conducted an analysis of the national cancer database (NCDB) and found decreased overall survival for patients who underwent radical nephroureterectomy greater than 120 days after diagnosis (26). In the era of neoadjuvant chemotherapy and immunotherapy, the theoretical risk of delayed radical nephroureterectomy must be weighed against the benefits of neoadjuvant therapy.

\section{Surgical approach}

Per the EAU guidelines, open radical nephroureterectomy with bladder cuff excision in the standard of care for highrisk UTUC (2). As new surgical techniques have been developed, laparoscopic nephroureterectomy has become a popular surgical intervention. The benefits of laparoscopy in urologic surgery are well described and include decreased blood loss, shorter hospital stay, and faster recovery. An early report on laparoscopic nephroureterectomy, however, noted trocar site recurrence (27), fueling concerns about oncologic safety of the laparoscopic technique. There were multiple reports of tumor seeding and port site metastasis, particularly in the early 2000s, as the technique was gaining in popularity (28). These early laparoscopic setbacks prompted a reinforcement of oncologic principles of laparoscopic surgery of the genitourinary tract, now codified in the EAU guidelines (2). The guidelines now specify that entry into the urinary tract should be avoided, direct contact between the instruments and tumor avoided, morcellation should not be utilized, the kidney and ureter (inclusive of bladder cuff) should be removed en bloc, and large tumors (T3/T4 or node or metastasis positive) tumors should be treated in an open fashion.

There is data demonstrating oncologic equivalency between the two methods (Table 1). Capitanio et al. evaluated nearly 1,300 patients in a multi-institutional data-set and showed comparable oncologic outcomes (30). These authors did note, however, that there was a selection bias in favor of laparoscopic nephroureterectomy patients having lower stage disease, less LVI, and more frequently solitary pelvicalyceal disease. Walton et al. evaluated 773 patients in a multi-institutional retrospective study and noted a recurrence-free survival of $74 \%$ for open nephroureterectomy and $63 \%$ for laparoscopic $(\mathrm{P}>0.1)$ and a CSS of $75 \%$ for both procedures (31). This study was noted, to have well matched cohorts. There is also level 1 evidence that laparoscopic nephroureterectomy can yield comparable oncologic outcomes. Simone et al. randomized 80 patients with no prior history of bladder urothelial carcinoma to radical open nephroureterectomy versus laparoscopic and found comparable CSS and MFS between the groups, except in patients with pT3 disease, in which case there was a CSS and MFS advantage to the open procedure (32). A recent meta-analysis confirms this finding that oncologic outcomes are poorer in patients with higher stage and risk disease (33). These authors hypothesize that this may be in part due to management of the bladder cuff, which is more difficult to excise in a standard fashion with pure laparoscopy, and technical difficulty associated with lymphadenectomy. The preponderance of evidence indicates that laparoscopy is likely safe in experienced hands on patients with lower risk disease, but open radical nephroureterectomy remains the standard of care in patients with bulky, high-risk disease.

The development of robotic surgery has created new questions regarding the feasibility and oncologic safety of robotic radical nephroureterectomy. The data on oncologic outcomes of the robotic procedure is less well elucidated. Lim et al. reported a case series of 32 patients who underwent robotic radical nephroureterectomy and reported a 5 -year CSS of $76 \%$ and RFS of $68 \%$, in line with prior reported literature of the open and laparoscopic procedures (34). Further single-series studies and meta-analyses have shown acceptable oncologic outcomes with respect to the robotic procedure $(35,36)$. No high-quality head-to-head studies exist examining the oncologic efficacy of robotic versus open or laparoscopic radical nephroureterectomy. This represents a significant gap in the current literature and further investigation is needed, as the robotic technique is 
Table 1 Comparison of outcomes of nephroureterectomy by technique

\begin{tabular}{|c|c|c|c|c|c|c|c|}
\hline Author & Time-frame & Technique & $\begin{array}{c}\text { Number of } \\
\text { patients }\end{array}$ & $\begin{array}{l}\text { dian follow-up } \\
\text { (months) }\end{array}$ & $\begin{array}{l}5-y r \text { recurrence free } \\
\text { survival }(\%)\end{array}$ & $\begin{array}{c}5 \text {-yr cancer specific } \\
\text { survival rate }(\%)\end{array}$ & Notes \\
\hline Capitanio et al. & 1987-2007 & ONU & 979 & 73 & 76.2 & 73.1 & $\begin{array}{l}\text { Patients who underwent } \\
\text { LNU were older but had } \\
\text { more favorable pathologic } \\
\text { staging and less LVI. After } \\
\text { adjustment for tumor stage, } \\
\text { no significant difference } \\
\text { in RFS or CSS based on } \\
\text { technique }\end{array}$ \\
\hline \multirow[t]{2}{*}{ Walton et al. } & 1987-2008 & ONU & 703 & 36 & 73.7 & 63.4 & \multirow[b]{2}{*}{$\begin{array}{l}\text { Patients who underwent } \\
\text { LNU were more likely } \\
\text { to have renal pelvis } \\
\text { tumors, received adjuvant } \\
\text { chemotherapy and higher } \\
\text { grade tumors. After } \\
\text { adjustments, no significant } \\
\text { difference in RFS or CSS } \\
\text { based on technique }\end{array}$} \\
\hline & & LNU & 70 & 17 & 75.4 & 75.2 & \\
\hline \multirow[t]{2}{*}{ Simone et al. } & 2003-2006 & ONU & 40 & 44 & 77.4 & 89.9 & \multirow[b]{2}{*}{$\begin{array}{l}\text { Prospective randomized } \\
\text { study demonstrating less } \\
\text { mean blood loss and time to } \\
\text { discharge with LNU. Overall, } \\
\text { no difference in RFS and } \\
\text { CSS but in patients with } \\
\geq \text { PT3 tumors, CSS and RFS } \\
\text { were in favor of ONU }\end{array}$} \\
\hline & & LNU & 40 & 44 & 72.5 & 79.8 & \\
\hline
\end{tabular}

ONU, open nephroureterectomy; LNU, laparoscopic nephroureterectomy; RANU, robotic assisted laparoscopic nephroureterectomy; LVI, lymphovascular invasion; RFS, recurrence free survival; CSS, cancer specific survival.

rising in popularity as urologists become more proficient with robotic techniques. In particular, it will be important to determine how robotic techniques have influenced management of the bladder cuff and lymphadenectomy, as these can both affect oncologic outcomes. It is possible that robotic approaches make these steps of the procedure technically easier and may affect practice patterns regarding bladder cuff management and lymphadenectomy.

\section{Management of the bladder cuff}

Historical reports of rates of ureteral stump recurrence of between $30 \%$ and $75 \%$ have driven the recommendation for bladder cuff excision during radical nephroureterectomy (37). It has been noted, however, that despite these historical numbers, the rates of formal bladder cuff excision in the literature remain low. One multiinstitutional retrospective analysis of nearly 1,300 patients who underwent nephroureterectomy demonstrated that only $41 \%$ of open and $50 \%$ of laparoscopic cases underwent formal bladder cuff excision, without effect on CSS (30). The decision to perform a bladder cuff excision in these patients, however, is not well delineated and it is possible that selection bias and unmatched cohorts played a role in this finding. Lughezzani et al. analyzed the SEER database and found increased cancer-specific mortality among pT3-4 and node-positive patients who did not undergo bladder cuff excision than those who did (38). Interestingly, 
patients with lower stage disease did not demonstrate this effect, leading the authors to conclude that a formal bladder cuff excision may not always be necessary. Some authors have postulated that these findings are likely due to data quality issues and further studies are needed to understand the true oncologic effect of bladder cuff excision (37). Guidelines continue to recommend bladder cuff excision in light of these gaps in knowledge.

The optimal approach to the management of the bladder cuff has also not been fully elucidated. Li et al. reported on the outcomes of 301 patients who underwent radical nephroureterectomy with one of three distal ureteral management techniques: intravesical incision, extravesical incision, and transurethral (endoscopic) incision with coagulation of the ureteral orifice and $1 \mathrm{~cm}$ cuff (39). These authors noted no differences in local or contralateral recurrence and no differences in RFS and CSS among these techniques. This study, however, was limited by its small sample size and short follow up. Xylinas et al. evaluated this question in a retrospective analysis of nearly 2,700 patients from 24 international institutions (40). These authors did not find any difference in CSS or OS among the three groups, but did note significantly higher rates of intravesical recurrence among those who underwent endoscopic bladder cuff management. They also noted that patients who underwent laparoscopic nephroureterectomy were more likely to receive endoscopic management of the bladder cuff. These data underscore the guideline recommendations that a bladder cuff excision should be performed at the time of nephroureterectomy and strongly suggest that an intravesical or extravesical approach should be utilized, although prospective studies could help guide this surgical decision. Some authors have speculated that the presence of an EndoWrist in robotic surgery may render these approaches more technically feasible and oncologically preferable (33).

\section{Role of lymphadenectomy}

The role of retroperitoneal lymphadenectomy at the time of radical nephroureterectomy remains controversial. Given the heterogeneity in lymphatic drainage along the course of the upper urinary tract, there is no agreed upon template for retroperitoneal lymphadenectomy at the time of radical nephroureterectomy, as well as lack of consensus indication for the procedure (2). Studies examining the oncologic necessity of lymphadenectomy are conflicting. Roscigno et al. (41) demonstrated in a multi-institutional cohort that node positive disease was a prognostic indicator of disease progression, as had been previously demonstrated in the literature (42). These authors also demonstrated that for patients with pT2-4 disease, unknown know nodal status $(\mathrm{pNx})$ demonstrated decreased 5-year CSS than $\mathrm{pN} 0$ disease. Lughezzani et al. evaluated the oncologic outcomes of approximately 1,800 patients without clinically apparent metastasis and noted no oncologic differences between $\mathrm{pN} 0$ and $\mathrm{pNx}$ patients (43). Further studies have indicated some benefit to lymphadenectomy, however. Zareba $\mathrm{et}$ al. conducted an analysis of the NCDB cohort and noted that among the pN0 subgroup, higher lymph node yield on lymphadenectomy was associated with improved all-cause mortality (44). The finding that increased lymph node yield improves CSS has been noted in a number of multiinstitutional studies (45). Not all authors have found this effect. In a small single-institutional series of 109 patients, Kondo et al. found no effect of node yield on oncologic outcomes, but did find oncological benefits with a greater extent of lymphadenectomy (46), suggesting that choice of template may be the most important factor in performing a lymphadenectomy at time of radical nephroureterectomy.

UTUC is notoriously difficult to stage and the question of patient selection for lymphadenectomy at time of surgery remains open. Patients with high-risk, highvolume, and clinically node-positive disease are likely to benefit. Prospective studies, however, are necessary to determine indications, ideal templates, and goal lymph node yields to obtain optimal oncologic adults. Until these data are known, it is not unreasonable to routinely perform lymphadenectomy at the time of nephroureterectomy given the possible oncologic benefits and improved staging information (45), particularly as it may relate to administration of adjuvant therapy.

\section{Intravesical chemotherapy}

Intravesical recurrence after nephroureterectomy has been demonstrated to be between $22 \%$ and $47 \%$ (47). Given known successes of post-transurethral resection of bladder tumor administration of intravesical therapy, investigators have taken interest in the role of intravesical chemotherapy following nephroureterectomy. O'Brien et al. conducted a prospective, randomized trial to evaluate the efficacy of single-dose intravesical mitomycin-C in a cohort of 140 patients (48). These authors found an absolute risk reduction of $11 \%$ at one year and relative risk reduction of $40 \%$. Ito et al. found similar one-year benefit with 
administration of intravesical pirarubicin within 48 hours of radical nephroureterectomy (49). Interestingly, there were no intravesical recurrences in year two of follow-up in the intravesical chemotherapy group, so the absolute risk reduction rose from $14.9 \%$ in year 1 to $25.3 \%$ at two years follow up. In light of this evidence, the EAU recommends postoperative instillation of chemotherapy after radical nephroureterectomy (2). In light of the promising results with use of gemcitabine for intravesical chemotherapy after TURBT (50), it is possible this will become an investigational agent for intravesical therapy in upper tract disease.

\section{Pathologic features}

Given the relatively low incidence of UTUC, much of the early literature regarding oncologic outcomes was derived from single center series often spanning several decades of patient data and non-standardized treatment paradigms (51-54). Batata et al. first described (55) 5-year survival data for patients undergoing surgical intervention for primary ureteral malignancy between 1947 and 1972. They noted a marked survival drop-off by pathologic stage of disease, with a $91 \% 5$-year survival for patients with submucosal invasion versus $43 \%$ for muscular invasion and $23 \%$ for extraureteral involvement and noted that nodal involvement was of particular prognostic significance. This early study, however, was limited by the fact that nearly half the cohort experienced prior or concomitant urothelial malignancies. This is notable, as only approximately $17 \%$ of UTUC present with concurrent bladder malignancy (2). Subsequent studies have attempted to control for such prognostic confounders, although large multi-institutional efforts have been most successful in doing so. Further, authors have sought to stratify out the features aside from stage that are predictive of mortality.

\section{Pathologic stage}

These early data have proven remarkably durable in more refined analyses of the literature. The UTUC collaboration data captured over 1,300 patients who underwent radical nephroureterectomy for UTUC. These data showed 5 -year cancer specific survival of $94 \%$ for pT0/Ta, $91 \%$ for $\mathrm{pT} 1,75 \%$ for $\mathrm{pT} 2,54 \%$ for $\mathrm{pT} 3$, and $12 \%$ for $\mathrm{pT} 4$ disease (4). With respect to tumor recurrence, these data showed a $92 \%, 89 \%, 71 \%, 48 \%$, and $4.7 \%$ RFS at 5 years, respectively. Numerous other studies have similarly demonstrated the importance of pathologic tumor stage in determining cancer-specific outcomes $(2,3,14,54,56)$ and tumor stage is incorporated into currently used predictive tools to determine patient prognosis.

\section{Grade}

Tumor grade is another critically important prognostic factor for UTUC. The importance of tumor grade is two-fold: first in being predictive of final pathology on nephroureterectomy and secondly in predicting long-term oncologic outcomes of nephroureterectomy. Clements et al. evaluated 238 cases in which patients underwent ureteroscopic biopsy and subsequent surgical treatment for UTUC (57). The authors noted that grade, not stage, was predictive of muscle invasion on final surgical pathology, with high grade disease on biopsy having a $60 \%$ positive predictive value for muscle invasive on final pathology. Therefore, ureteroscopic grade was actually a more important predictor of pathologic stage, which as previously discussed, is one of the most important determinants of cancer and recurrence-free survival. Other studies evaluating prognostic predictors have further demonstrated that grade on final nephroureterectomy pathology is also an important determinant of CSS $(4,14,54,56)$. Margulis et al. in their analysis of the UTUC collaborative data found that patients with low grade disease on nephroureterectomy had an $89 \%$ cancer specific survival at 5 years versus $63 \%$ CSS for high grade disease. Similarly, these authors found an RFS of $88 \%$ versus $57 \%$ at 5 years for low and high grade disease, respectively.

\section{Margins}

Surgical margins are a less-thoroughly studied pathologic finding with regard to oncologic outcomes of radical nephroureterectomy, although appear to be quite important. Colin et al. evaluated the role of positive surgical margins on cancer-specific and metastasis-free survival (58). The presence of positive surgical margins decreased 5-year cancer specific survival from $83 \%$ to $59 \%$ and metastasisfree survival from $79 \%$ to $52 \%$ and found positive margins to be an independent predictor of metastasis-free survival. These authors also found that ureteral tumors, particularly multifocal ones, had a higher incidence of positive surgical margins than pelvicalyceal UTUC. Many of these positive margins were found in the ureter and underscore the importance of proper surgical technique, given the profound effect of positive margins on survival. 


\section{Tumor location}

The location of ureteral tumors and its effect on oncologic outcomes has been studied by multiple other authors and remains a matter of some controversy. Initial single-center series (59) and multi-institutional studies did not show a clear effect of tumor multifocality on oncologic outcomes (60). Subsequent studies have demonstrated a relationship between tumor multifocality and CSS. Ouzzane et al. demonstrated in a series of 609 patients in a national collaborative dataset that the 5 -year CSS was $87 \%$ with renal pelvis tumors, $69 \%$ for solitary ureteral tumors, and $57 \%$ for multifocal tumors (defined as in the renal pelvis and ureter) (61). Similarly, Chromecki et al., in the largest series examining tumor multifocality including nearly 2,500 patients, demonstrated that in patients treated with radical nephroureterectomy there was decreased cancer-specific and recurrence-free survival for patients with multifocal disease (62). The effect was noted to be slightly more attenuated than noted in the prior study, with 5 -year CSS in the multifocal group at $68 \%$ vs. $73 \%$ for the unifocal group, perhaps owing to varying definitions of multifocality. The largest multi-institutional data on this subject by Cha et al., however, did not demonstrate tumor location to be an independent predictor of CSS and RFS (63). Given the largest multi-institutional cohorts have found, at best, a modest improvement in CSS and RFS depending on tumor location, its prognostic use of location is in question. Multifocality persists, however, as a useful prognostic indicator.

\section{Lymphovascular invasion (LVI)}

LVI is noted in nearly one-quarter of all radical nephroureterectomy specimens $(64,65)$. The presence of LVI on radical nephroureterectomy specimens is an important predictor of RFS and CSS. Kikuchi et al. found a 5 -year CSS of 79\% without LVI versus $47 \%$ with LVI (64). These authors noted a recurrence-free survival difference as well at 5 years, with $77 \%$ for those without LVI and $44 \%$ for patients with LVI. These differences were noted to be present even when excluding patients with node-positive disease on final pathology. The predictive value of LVI on oncologic outcomes has been demonstrated in subsequent multi-institutional cohort studies $(4,65)$.

\section{Carcinoma in-situ (CIS)}

The presence of organ-confined and bladder CIS have also been evaluated as predictors of oncologic outcomes. Otto et al. evaluated a cohort of 772 neoadjuvant-naïve patients who underwent radical nephroureterectomy and found a 5 -year CSS and RFS difference between the groups (66). These authors noted concomitant CIS in approximately $11 \%$ of cases and found that the presence of concomitant CIS increased with more advanced $\mathrm{T}$ stage, higher tumor grade, and presence of LVI. Nevertheless, CIS held up as an independent predictor of survival on multivariate analysis. A subsequent analysis has demonstrated the concomitant CIS confers a worse oncologic prognosis than CIS alone and speculate that this could represent a different biological entity entirely (67). Wheat et al. evaluated concomitant CIS in a cohort of nearly 1,400 patients and found a far greater incidence of concomitant CIS of $26.7 \%$, but similar improvements in CSS and RFS in cases of organconfined disease (68). The role of bladder CIS has also been investigated, given $2 \%$ to $7 \%$ of patients with primary bladder urothelial cancer develop upper tract tumors (69). Youssef et al. found that the presence of prior bladder CIS decreased 5 -year CSS from $75 \%$ to $59 \%$ and RFS from $71 \%$ to $53 \%$ (70), which subsequently held up on multivariable analysis.

\section{Tumor architecture}

Macroscopic tumor architecture has also been implicated as a prognostic feature of aggressive UTUC. Remzi et al. noted in a multi-institutional cohort of nearly 1,400 patients that approximately one-quarter of patients harbored sessile tumors and the presence of this feature was an independent predictor of cancer-specific and recurrence-free survival and improved the diagnostic accuracy of previously utilized prognostic nomograms (71). A number of such prognostic models have been developed using previously demonstrated predictors of UTUC CSS and RFS. Cha et al. created and studied the most widely accepted prognostic nomogram for these factors based on a sample of 2,244 patients from the UTUC collaborative group data (63). As with previous studies, these authors found $\mathrm{T}$ stage, lymph node metastasis, LVI, sessile tumor architecture, and concomitant CIS associated with RFS on multivariable analysis and all of these features associated with CSS except CIS on multivariable analysis. Interestingly, grade was not an independent predictor on multivariable analysis, perhaps due to the fact that $80 \%$ of the cohort harbored high grade disease. Also, tumor location was not noted to be predictive and included in this nomogram. These authors were able to predict RFS and CSS with $76.8 \%$ an $81.5 \%$ accuracy, respectively, with inclusion of age, $\mathrm{T}$ stage, 
grade, $\mathrm{N}$ classification, LVI, architecture, and concomitant CIS into their nomogram. Given available evidence, these features are best considered when counseling patients about prognosis of UTUC after radical nephroureterectomy. Some authors also include preoperative hydronephrosis into predictive tools, as this has also been shown to correlate with pathologic stage, grade, and nodal metastasis and independently predict CSS and RFS $(14,72,73)$. The use of such predictive tools in practice, however, is not well defined and refinements continue to be made to these nomograms as more pathologic, serum, and genetic predictors of disease progression are identified.

\section{Conclusions}

Oncologic outcomes of nephroureterectomy have been remarkably stable over the past several decades. Progress has been made in the past decade at more accurately determining prognostic markers of disease progression and will continue to improve as understanding of tumor biology increases. The role of multi-institutional collaboration in these efforts cannot be understated, as the relative rarity of the disease makes single-center studies of UTUC very difficult to conduct and often underpowered. Advances in technology, such as the rise of laparoscopic and robotic approaches to nephroureterectomy, have altered practice, although do not appear to have improved oncologic outcomes and may, at times, prove detrimental. Further studies are certainly indicated to optimize $21^{\text {st }}$ century surgical techniques and ensure that they achieve at least comparable oncologic control as $20^{\text {th }}$ century procedures.

The use of perioperative chemotherapy and immunotherapy appear best poised to augment the efficacy of surgical resection. Perhaps the most significant oncologic improvement in the treatment of UTUC was demonstrated with the POUT trial, in which adjuvant chemotherapy improved RFS from $50 \%$ to $70 \%$ at two years (74). Further promise has been demonstrated with ECOG 8141 and administration of neoadjuvant chemotherapy, in which a complete pathologic response was noted in $14 \%$ of patients who received this therapy (75). As use of these treatment modalities is expanded, as well as the use of immunotherapy, which has shown promise in the treatment of bladder urothelial malignancy (76), there will be an increased need for collaboration to study nephroureterectomy outcomes and potentially re-define the patients for whom this intervention is indicated. It is unlikely that nephroureterectomy will become obsolete in the era of immunotherapy and targeted biologic agents and there is great reason to be hopeful that the procedure will become more effective and curative in the future.

\section{Acknowledgments}

Funding: None.

\section{Footnote}

Provenance and Peer Review: This article was commissioned by the Guest Editors (John J. Knoedler and Jay D. Raman) for the series "Upper-Tract Urothelial Carcinoma: Current State and Future Directions" published in Translational Andrology and Urology. The article was sent for external peer review organized by the Guest Editors and the editorial office.

Conflicts of Interest: The series "Upper-Tract Urothelial Carcinoma: Current State and Future Directions" was commissioned by the editorial office without any funding or sponsorship. The authors have no other conflicts of interest to declare.

Ethical Statement: The authors are accountable for all aspects of the work in ensuring that questions related to the accuracy or integrity of any part of the work are appropriately investigated and resolved.

Open Access Statement: This is an Open Access article distributed in accordance with the Creative Commons Attribution-NonCommercial-NoDerivs 4.0 International License (CC BY-NC-ND 4.0), which permits the noncommercial replication and distribution of the article with the strict proviso that no changes or edits are made and the original work is properly cited (including links to both the formal publication through the relevant DOI and the license). See: https://creativecommons.org/licenses/by-nc-nd/4.0/.

\section{References}

1. Siegel RL, Miller KD, Jemal A. Cancer statistics, 2019. CA Cancer J Clin 2019;69:7-34.

2. Rouprêt M, Babjuk M, Comperat E, et al. European Association of Urology Guidelines on Upper Urinary Tract Urothelial Carcinoma: 2017 Update. Eur Urol 2018;73:111-22.

3. Munoz JJ, Ellison LM. Upper tract urothelial neoplasms: incidence and survival during the last 2 decades. J Urol 2000;164:1523-5.

4. Margulis V, Shariat SF, Matin SF, et al. Outcomes of 
radical nephroureterectomy: a series from the Upper Tract Urothelial Carcinoma Collaboration. Cancer 2009; 115:1224-33.

5. Mazeman E. Tumors of the upper urinary tract (calices, pelvis, ureter). J Chir (Paris) 1974;107:473-98.

6. Johnson DE, Babaian RJ. Conservative surgical management for noninvasive distal ureteral carcinoma. Urology 1979;13:365-7.

7. Zungri E, Chechile G, Algaba F, et al. Treatment of transitional cell carcinoma of the ureter: is the controversy justified? Eur Urol 1990;17:276-80.

8. Colin P, Ouzzane A, Pignot G, et al. Comparison of oncological outcomes after segmental ureterectomy or radical nephroureterectomy in urothelial carcinomas of the upper urinary tract: results from a large French multicentre study. BJU Int 2012;110:1134-41.

9. Fang D, Seisen T, Yang K, et al. A systematic review and meta-analysis of oncological and renal function outcomes obtained after segmental ureterectomy versus radical nephroureterectomy for upper tract urothelial carcinoma. Eur J Surg Oncol 2016;42:1625-35.

10. Lughezzani G, Jeldres C, Isbarn H, et al. Nephroureterectomy and segmental ureterectomy in the treatment of invasive upper tract urothelial carcinoma: a population-based study of 2299 patients. Eur J Cancer 2009;45:3291-7.

11. Bagrodia A, Kuehhas FE, Gayed BA, et al. Comparative analysis of oncologic outcomes of partial ureterectomy vs radical nephroureterectomy in upper tract urothelial carcinoma. Urology 2013;81:972-7.

12. Strong DW, Pearse HD, Tank ES Jr, et al. The ureteral stump after nephroureterectomy. J Urol 1976;115:654-5.

13. Shariat SF, Godoy G, Lotan Y, et al. Advanced patient age is associated with inferior cancer-specific survival after radical nephroureterectomy. BJU Int 2010;105:1672-7.

14. Lughezzani G, Burger M, Margulis V, et al. Prognostic factors in upper urinary tract urothelial carcinomas: a comprehensive review of the current literature. Eur Urol 2012;62:100-14.

15. Shariat SF, Sfakianos JP, Droller MJ, et al. The effect of age and gender on bladder cancer: a critical review of the literature. BJU Int 2010;105:300-8.

16. Lughezzani G, Sun M, Perrotte P, et al. Gender-related differences in patients with stage I to III upper tract urothelial carcinoma: results from the Surveillance, Epidemiology, and End Results database. Urology 2010;75:321-7.

17. Shariat SF, Favaretto RL, Gupta A, et al. Gender differences in radical nephroureterectomy for upper tract urothelial carcinoma. World J Urol 2011;29:481-6.

18. Raman JD, Messer J, Sielatycki JA, et al. Incidence and survival of patients with carcinoma of the ureter and renal pelvis in the USA, 1973-2005. BJU Int 2011;107:1059-64.

19. Rink M, Xylinas E, Margulis V, et al. Impact of smoking on oncologic outcomes of upper tract urothelial carcinoma after radical nephroureterectomy. Eur Urol 2013;63:1082-90.

20. Xylinas E, Kluth LA, Rieken M, et al. Impact of smoking status and cumulative exposure on intravesical recurrence of upper tract urothelial carcinoma after radical nephroureterectomy. BJU Int 2014;114:56-61.

21. Ehdaie B, Chromecki TF, Lee RK, et al. Obesity adversely impacts disease specific outcomes in patients with upper tract urothelial carcinoma. J Urol 2011;186:66-72.

22. Dabi Y, El Mrini M, Duquesnes I, et al. Impact of body mass index on the oncological outcomes of patients treated with radical nephroureterectomy for upper tract urothelial carcinoma. World J Urol 2018;36:65-71.

23. Boorjian $\mathrm{S}, \mathrm{Ng} \mathrm{C}$, Munver R, et al. Impact of delay to nephroureterectomy for patients undergoing ureteroscopic biopsy and laser tumor ablation of upper tract transitional cell carcinoma. Urology 2005;66:283-7.

24. Sankin A, Tin AL, Mano R, et al. Impact of Ureteroscopy Before Nephroureterectomy for Upper Tract Urothelial Carcinoma on Oncologic Outcomes. Urology 2016;94:148-53.

25. Sundi D, Svatek RS, Margulis V, et al. Upper tract urothelial carcinoma: impact of time to surgery. Urol Oncol 2012;30:266-72.

26. Xia L, Taylor BL, Pulido JE, et al. Impact of surgical waiting time on survival in patients with upper tract urothelial carcinoma: A national cancer database study. Urol Oncol 2018;36:10.e15-10.e22.

27. Ong AM, Bhayani SB, Pavlovich CP. Trocar site recurrence after laparoscopic nephroureterectomy. J Urol 2003;170:1301.

28. Rouprêt M, Smyth G, Irani J, et al. Oncological risk of laparoscopic surgery in urothelial carcinomas. World J Urol 2009;27:81-8.

29. De Groote R, Decaestecker K, Larcher A, et al. Robotassisted nephroureterectomy for upper tract urothelial carcinoma: results from three high-volume robotic surgery institutions. J Robot Surg 2020;14:211-9.

30. Capitanio U, Shariat SF, Isbarn H, et al. Comparison of oncologic outcomes for open and laparoscopic nephroureterectomy: a multi-institutional analysis of 1249 cases. Eur Urol 2009;56:1-9.

31. Walton TJ, Novara G, Matsumoto K, et al. Oncological outcomes after laparoscopic and open radical 
nephroureterectomy: results from an international cohort. BJU Int 2011;108:406-12.

32. Simone G, Papalia R, Guaglianone S, et al. Laparoscopic versus open nephroureterectomy: perioperative and oncologic outcomes from a randomised prospective study. Eur Urol 2009;56:520-6.

33. Peyronnet B, Seisen T, Dominguez-Escrig JL, et al. Oncological Outcomes of Laparoscopic Nephroureterectomy Versus Open Radical Nephroureterectomy for Upper Tract Urothelial Carcinoma: An European Association of Urology Guidelines Systematic Review. Eur Urol Focus 2019;5:205-23.

34. Lim SK, Shin TY, Kim KH, et al. Intermediateterm outcomes of robot-assisted laparoscopic nephroureterectomy in upper urinary tract urothelial carcinoma. Clin Genitourin Cancer 2013;11:515-21.

35. Aboumohamed AA, Krane LS, Hemal AK. Oncologic Outcomes Following Robot-Assisted Laparoscopic Nephroureterectomy with Bladder Cuff Excision for Upper Tract Urothelial Carcinoma. J Urol 2015;194:1561-6.

36. Mullen E, Ahmed K, Challacombe B. Systematic review of open versus laparoscopic versus robot-assisted nephroureterectomy. Rev Urol 2017;19:32-43.

37. Zlotta AR. Should urologists always perform a bladder cuff resection during nephroureterectomy, and which method should they use? Eur Urol 2010;57:970-2.

38. Lughezzani G, Sun M, Perrotte P, et al. Should bladder cuff excision remain the standard of care at nephroureterectomy in patients with urothelial carcinoma of the renal pelvis? A population-based study. Eur Urol 2010;57:956-62.

39. Li WM, Shen JT, Li CC, et al. Oncologic outcomes following three different approaches to the distal ureter and bladder cuff in nephroureterectomy for primary upper urinary tract urothelial carcinoma. Eur Urol 2010;57:963-9.

40. Xylinas E, Rink M, Cha EK, et al. Impact of distal ureter management on oncologic outcomes following radical nephroureterectomy for upper tract urothelial carcinoma. Eur Urol 2014;65:210-7.

41. Roscigno M, Shariat SF, Margulis V, et al. Impact of lymph node dissection on cancer specific survival in patients with upper tract urothelial carcinoma treated with radical nephroureterectomy. J Urol 2009;181:2482-9.

42. Komatsu H, Tanabe N, Kubodera S, et al. The role of lymphadenectomy in the treatment of transitional cell carcinoma of the upper urinary tract. J Urol 1997;157:1622-4.

43. Lughezzani G, Jeldres C, Isbarn H, et al. A critical appraisal of the value of lymph node dissection at nephroureterectomy for upper tract urothelial carcinoma. Urology 2010;75:118-24.

44. Zareba P, Rosenzweig B, Winer AG, et al. Association between lymph node yield and survival among patients undergoing radical nephroureterectomy for urothelial carcinoma of the upper tract. Cancer 2017;123:1741-50.

45. Seisen T, Shariat SF, Cussenot O, et al. Contemporary role of lymph node dissection at the time of radical nephroureterectomy for upper tract urothelial carcinoma. World J Urol 2017;35:535-48.

46. Kondo T, Hashimoto Y, Kobayashi H, et al. Templatebased lymphadenectomy in urothelial carcinoma of the upper urinary tract: impact on patient survival. Int J Urol 2010;17:848-54.

47. Seisen T, Granger B, Colin P, et al. A Systematic Review and Meta-analysis of Clinicopathologic Factors Linked to Intravesical Recurrence After Radical Nephroureterectomy to Treat Upper Tract Urothelial Carcinoma. Eur Urol 2015;67:1122-33.

48. O'Brien T, Ray E, Singh R, et al. Prevention of bladder tumours after nephroureterectomy for primary upper urinary tract urothelial carcinoma: a prospective, multicentre, randomised clinical trial of a single postoperative intravesical dose of mitomycin $\mathrm{C}$ (the ODMIT-C Trial). Eur Urol 2011;60:703-10.

49. Ito A, Shintaku I, Satoh M, et al. Prospective randomized phase II trial of a single early intravesical instillation of pirarubicin (THP) in the prevention of bladder recurrence after nephroureterectomy for upper urinary tract urothelial carcinoma: the THP Monotherapy Study Group Trial. J Clin Oncol 2013;31:1422-7.

50. Messing EM, Tangen CM, Lerner SP, et al. Effect of Intravesical Instillation of Gemcitabine vs Saline Immediately Following Resection of Suspected LowGrade Non-Muscle-Invasive Bladder Cancer on Tumor Recurrence: SWOG S0337 Randomized Clinical Trial. JAMA 2018;319:1880-8.

51. Guinan P, Vogelzang NJ, Randazzo R, et al. Renal pelvic cancer: a review of 611 patients treated in Illinois 1975 1985. Cancer Incidence and End Results Committee. Urology 1992;40:393-9.

52. Murphy DM, Zincke H, Furlow WL. Primary grade 1 transitional cell carcinoma of the renal pelvis and ureter. J Urol 1980;123:629-31.

53. Bloom NA, Vidone RA, Lytton B. Primary carcinoma of the ureter: a report of 102 new cases. J Urol 1970;103:590-8.

54. Hall MC, Womack S, Sagalowsky AI, et al. Prognostic factors, recurrence, and survival in transitional cell 
carcinoma of the upper urinary tract: a 30-year experience in 252 patients. Urology 1998;52:594-601.

55. Batata MA, Whitmore WF, Hilaris BS, et al. Primary carcinoma of the ureter: a prognostic study. Cancer 1975;35:1626-32.

56. Novara G, De Marco V, Gottardo F, et al. Independent predictors of cancer-specific survival in transitional cell carcinoma of the upper urinary tract: multi-institutional dataset from 3 European centers. Cancer 2007;110:1715-22.

57. Clements T, Messer JC, Terrell JD, et al. High-grade ureteroscopic biopsy is associated with advanced pathology of upper-tract urothelial carcinoma tumors at definitive surgical resection. J Endourol 2012;26:398-402.

58. Colin P, Ouzzane A, Yates DR, et al. Influence of positive surgical margin status after radical nephroureterectomy on upper urinary tract urothelial carcinoma survival. Ann Surg Oncol 2012;19:3613-20.

59. Favaretto RL, Shariat SF, Chade DC, et al. The effect of tumor location on prognosis in patients treated with radical nephroureterectomy at Memorial Sloan-Kettering Cancer Center. Eur Urol 2010;58:574-80.

60. Raman JD, Ng CK, Scherr DS, et al. Impact of tumor location on prognosis for patients with upper tract urothelial carcinoma managed by radical nephroureterectomy. Eur Urol 2010;57:1072-9.

61. Ouzzane A, Colin P, Xylinas E, et al. Ureteral and multifocal tumours have worse prognosis than renal pelvic tumours in urothelial carcinoma of the upper urinary tract treated by nephroureterectomy. Eur Urol 2011;60:1258-65.

62. Chromecki TF, Cha EK, Fajkovic H, et al. The impact of tumor multifocality on outcomes in patients treated with radical nephroureterectomy. Eur Urol 2012;61:245-53.

63. Cha EK, Shariat SF, Kormaksson M, et al. Predicting clinical outcomes after radical nephroureterectomy for upper tract urothelial carcinoma. Eur Urol 2012;61:818-25.

64. Kikuchi E, Margulis V, Karakiewicz PI, et al. Lymphovascular invasion predicts clinical outcomes in patients with node-negative upper tract urothelial carcinoma. J Clin Oncol 2009;27:612-8.

65. Novara G, Matsumoto K, Kassouf W, et al. Prognostic role of lymphovascular invasion in patients with urothelial carcinoma of the upper urinary tract: an international validation study. Eur Urol 2010;57:1064-71.

66. Otto W, Shariat SF, Fritsche HM, et al. Concomitant carcinoma in situ as an independent prognostic parameter for recurrence and survival in upper tract urothelial carcinoma: a multicenter analysis of 772 patients. World J Urol 2011;29:487-94.

67. Inamoto $\mathrm{T}$, Matsuyama $\mathrm{H}$, Ibuki $\mathrm{N}$, et al. Biological Behavior and Long-Term Outcomes of Carcinoma
In Situ in Upper Urinary Tract Managed by Radical Nephroureterectomy. J Urol 2018;199:933-9.

68. Wheat JC, Weizer AZ, Wolf JS Jr, et al. Concomitant carcinoma in situ is a feature of aggressive disease in patients with organ confined urothelial carcinoma following radical nephroureterectomy. Urol Oncol 2012;30:252-8.

69. Wright JL, Hotaling J, Porter MP. Predictors of upper tract urothelial cell carcinoma after primary bladder cancer: a population based analysis. J Urol 2009;181:10359; discussion 1039.

70. Youssef RF, Shariat SF, Lotan Y, et al. Prognostic effect of urinary bladder carcinoma in situ on clinical outcome of subsequent upper tract urothelial carcinoma. Urology 2011;77:861-6.

71. Remzi M, Haitel A, Margulis V, et al. Tumour architecture is an independent predictor of outcomes after nephroureterectomy: a multi-institutional analysis of 1363 patients. BJU Int 2009;103:307-11.

72. Cho KS, Hong SJ, Cho NH, et al. Grade of hydronephrosis and tumor diameter as preoperative prognostic factors in ureteral transitional cell carcinoma. Urology 2007;70:662-6.

73. Ng CK, Shariat SF, Lucas SM, et al. Does the presence of hydronephrosis on preoperative axial CT imaging predict worse outcomes for patients undergoing nephroureterectomy for upper-tract urothelial carcinoma? Urol Oncol 2011;29:27-32.

74. Birtle AJ, Chester JD, Jones RJ, et al. Results of POUT: A phase III randomised trial of perioperative chemotherapy versus surveillance in upper tract urothelial cancer (UTUC). J Clin Oncol 2018;36:407.

75. Hoffman-Censits J, Puligandla M, Trabulsi E, et al. LBA26 phase II trial of neoadjuvant chemotherapy followed by extirpative surgery for patients with high grade upper tract urothelial carcinoma (HG UTUC): results from ECOGACRIN 8141. J Urol 2018;199:e1166-7.

76. Necchi A, Anichini A, Raggi D, et al. Pembrolizumab as Neoadjuvant Therapy Before Radical Cystectomy in Patients With Muscle-Invasive Urothelial Bladder Carcinoma (PURE-01): An Open-Label, SingleArm, Phase II Study. J Clin Oncol 2018. doi: 10.1200/ JCO.18.01148.

Cite this article as: Kenigsberg AP, Meng X, Ghandour R, Margulis V. Oncologic outcomes of radical nephroureterectomy (RNU). Transl Androl Urol 2020;9(4):1841-1852. doi: 10.21037/ tau.2019.12.29 